\title{
Endovascular Thrombectomy for Distal Occlusion Using a Semi-Deployed Stentriever: Report of 2 Cases and Technical Note
}

\author{
Yue Wan, $\mathrm{MD}^{1}$, I-Hsiao Yang, $\mathrm{MD}^{2,3}$, Emanuele Orru, $\mathrm{MD}^{3}$, Timo Krings, $\mathrm{MD}^{3}$, \\ Anderson Chun On Tsang, MBBS ${ }^{3,4}$ \\ ${ }^{1}$ Department of Neurology, The Third People's Hospital of Hubei, Wuhan, China \\ ${ }^{2}$ Department of Medical Imaging, Kaohsiung Medical University Hospital, Kaohsiung, Taiwan \\ ${ }^{3}$ Division of Neuroradiology, Joint Department of Medical Imaging, Toronto Western Hospital, Toronto, Canada \\ ${ }^{4}$ Division of Neurosurgery, Department of Surgery, The University of Hong Kong, Hong Kong
}

\begin{abstract}
Distal intracranial occlusions can sometimes cause significant neurological deficits. Endovascular thrombectomy in these vessels may improve outcome but carry a higher risk of haemorrhagic complications due to the small calibre and tortuosity of the target vessel. We report two cases of isolated M2/3 artery occlusion causing dense hemiplegia that was successfully treated with stent retrieval thrombectomy. A "semi-deployment technique" of a $3 \mathrm{~mm}$ stentriever was employed at the M2/3 bifurcation of the middle cerebral artery. Partial stent unsheathing allowed adequate clot engagement while avoiding excessive tension by the stent metal struts along the tortuous course of a distal vessel. Complete revascularization was achieved after firstpass of the stent retriever without complication, resulting in good clinical outcome in both cases. The described semi-deployment technique reduces the radial and tractional force exerted by the stentreiver on small branches, and may reduce the risk of vessel laceration or dissection in distal vessel thrombectomy.
\end{abstract}

Key Words: Thrombectomy; Stroke; Endovascular procedures

\section{INTRODUCTION}

Mechanical thrombectomy is the well-accepted standard of care for acute large vessel occlusions in the internal carotid artery (ICA) and in the M1 segment of the middle cerebral artery (MCA). ${ }^{1}$ The safety and efficacy of thrombectomy in more distal intracranial branches are on the other hand less clear. Recent observational studies showed promising results for distal M2 and M3 occlusions treated with stent retrieval thrombec- tomy. ${ }^{2}$ Occluded distal branches that supply areas of functionally significant parenchyma could potentially benefit from endovascular thrombectomy in selected cases, but, feasibility of this technique is limited by the distal thrombus location and by the tortuosity and potential fragility of these small vessels, hence harbouring a presumed higher risk of complications.

\section{Correspondence to:} Anderson Chun On Tsang, MBBS Division of Neurosurgery, Queen Mary Hospital, Room 701 Administrative Block, 102 Pokfulam Road, Hong Kong Tel: +852 22553368

Fax: +85228184350

E-mail: acotsang@hku.hk

Received: June 26, 2019

Revised: August 3, 2019

Accepted: August 6, 2019
Copyright $\odot 2019$ Korean Society of Interventional Neuroradiology

This is an Open Access article distributed under the terms of the Creative Commons Attribution Non-Commercial License (http://creativecommons.org/licenses/by-nc/3.0) which permits unrestricted non-commercial use, distribution, and reproduction in any medium, provided the original work is properly cited. 


\section{MATERIALS AND METHODS}

\section{Endovascular technique}

After confirmation of the vessel occlusion at the M2/3 segment of MCA by computed tomography (CT) and selective catheter angiography (Fig. 1), a Flowgate balloon guiding catheter (Stryker, Kalamazoo, MI, USA) was placed in the distal cervical ICA and a Trevo Pro 14 (Stryker) microcatheter was then navigated past the occlusion over a Synchro 14 (Stryker) micro guidewire. In light of the small calibre and tortuosity of the occluded vessel, a small Trevo XP ProVue $3 \times 20 \mathrm{~mm}$ stent retriever (Stryker) was only partially deployed within the thrombus, leaving half of the stent length sheathed inside the microcatheter (Fig. 2A, B). The stent was kept open for 5 minutes for thrombus engagement and then retrieved under proximal flow reversal at the balloon guiding catheter, resulting in revascularization of the affected MCA territory (Fig. 2C, D).

\section{Illustrative cases}

\section{Case 1}

A 68-year-old male patient presented with acute left hemiplegia 2 hours before admission. Due to the left leg weakness, the patient fell and suffered a subtrochanteric fracture of his left femur. The initial National Institute of Health Stroke Scale (NIHSS) was 8. There was no intracranial haemorrhage and the Alberta Stroke Program Early CT Score was 10. A $\mathrm{CT}$-angiogram revealed a $7 \mathrm{~mm}$-long filling defect in the superior division ( $M 2$ trunk) of right MCA, with a modified collateral score of 3 (Fig. 1A, B). ${ }^{3}$ Despite the early presentation, intravenous thrombolysis (IV) was contraindicated due to the long bone fracture. The patient was then referred for endovascular thrombectomy.

An initial ICA angiogram demonstrated that the M2 thrombus had migrated distally into the origin of right precentral artery (M3), a vessel measuring $1.6 \mathrm{~mm}$ in diameter (Fig 1C, D). Considering the functional eloquence of the motor cortex supplied by this occluded branch, it was decided to attempt revascularization of this branch. Using a $3 \mathrm{~mm}$ stent-retriever and the semi-deployment technique, modified treatment in cerebral infarction ( $\mathrm{mTICl}) 3$ revascularization of the affected MCA territory was achieved (Fig. 2C, D). The groin puncture-to-reperfusion time was within 35 minutes.

The NIHSS improved from 8 to 2 in the first 24 hours after treatment, with resolution of the left hemiparesis. The patient underwent uneventful fixation of the left femoral fracture and was discharged to home within 1 week. A follow-up magnetic resonance imaging performed 3 days after thrombectomy showed patent MCA branches, a few scattered small foci of restricted diffusion in the right hemisphere suggestive of small distal emboli without evidence of large regional infarctions, and no hemorrhagic complications (Fig. 2E, F). The modified Rankin scale at discharge was 1.

\section{Case 2}

A 71-year-old man with left carotid bifurcation stenosis was admitted for acute right hemiplegia. The initial CT angiogram and subsequent catheter angiogram confirmed occlusion of the inferior $\mathrm{M} 2$ territory, obstructing the precentral artery (Fig. 3A, B). mTICI 3 revascularization was achieved with the semi-deployment of a Trevo XP ProVue $3 \times 20 \mathrm{~mm}$ stent retriever (Fig. 3C, D). The left cervical carotid artery was subsequently treated with stenting and angioplasty (Fig. 3E, F).
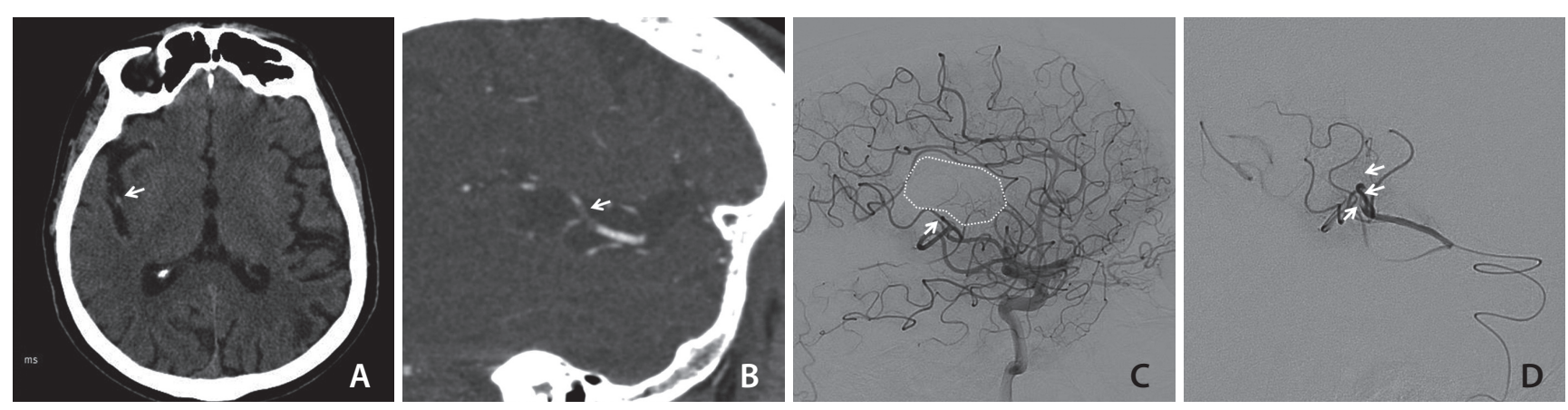

Fig. 1. (A) Non-contrast $C T$ brain showing hyperdense MCA thrombus in the M2/3 segment at the Sylvian fissure and (B) contrast CT angiogram with sagittal reconstruction demonstrating the filling defect at the M2/3 bifurcation of the opercular branch of MCA (arrow). (C) Lateral projection right ICA angiogram and (D) selective microcatheter angiogram from right precentral artery demonstrating the M3 precentral artery occlusion (arrows) and perfusion defect in the Rolandic area (dotted circle). CT, computed tomography; MCA, middle cerebral artery; ICA, internal carotid artery. 
The modified Rankin scale at discharge was 1.

\section{DISCUSSION}

Depending on the "eloquence" of the supplied territory, small cerebral vessel occlusions can lead to severe neurological deficits. While IV with tissue plasminogen activator (tPA) remains the standard of care, its use is frequently limited by the short therapeutic time window and by multiple contraindications, such as the long bone fracture in our first patient.

Endovascular mechanical thrombectomy provides rapid recanalization and improves the neurological outcome in large vessel occlusions of the ICA and M1 segment of MCA. This technique has been increasingly applied to M2 and M3 occlusions, but its benefits over medical treatment with IV tPA and its safety profile have not been clearly demonstrat- ed. In particular, the risk of vessel perforation and of haemorrhagic complications remains a concern. ${ }^{5}$

Haussen et al. ${ }^{5}$ reported four cases of M3 occlusions treated with thrombectomy with a $3 \mathrm{~mm}$ Trevo stentriever. Two of these patients suffered from intracranial haemorrhage after mechanical thrombectomy that led to poor outcomes. This relatively high rate of haemorrhagic complications may in part be due to the radial and tractional force exerted by the stent struts on these small and often tortuous vessels during retrieval of the device, resulting in perforator or venous avulsion or vessel laceration.

Using in-vitro models, Machi et al. ${ }^{6}$, demonstrated that the radial pressure $\left(R_{N}\right)$ exerted by stent retrievers on the vessel wall is inversely proportional to the length of the deployed stent. This was expressed by the formula $R_{N}=F /(2 \pi r L \mu)$, where $r$ is the internal radius of the blood vessel, $L$ is the length of the deployed stent and $\mu$ is the friction coefficient. ${ }^{6}$
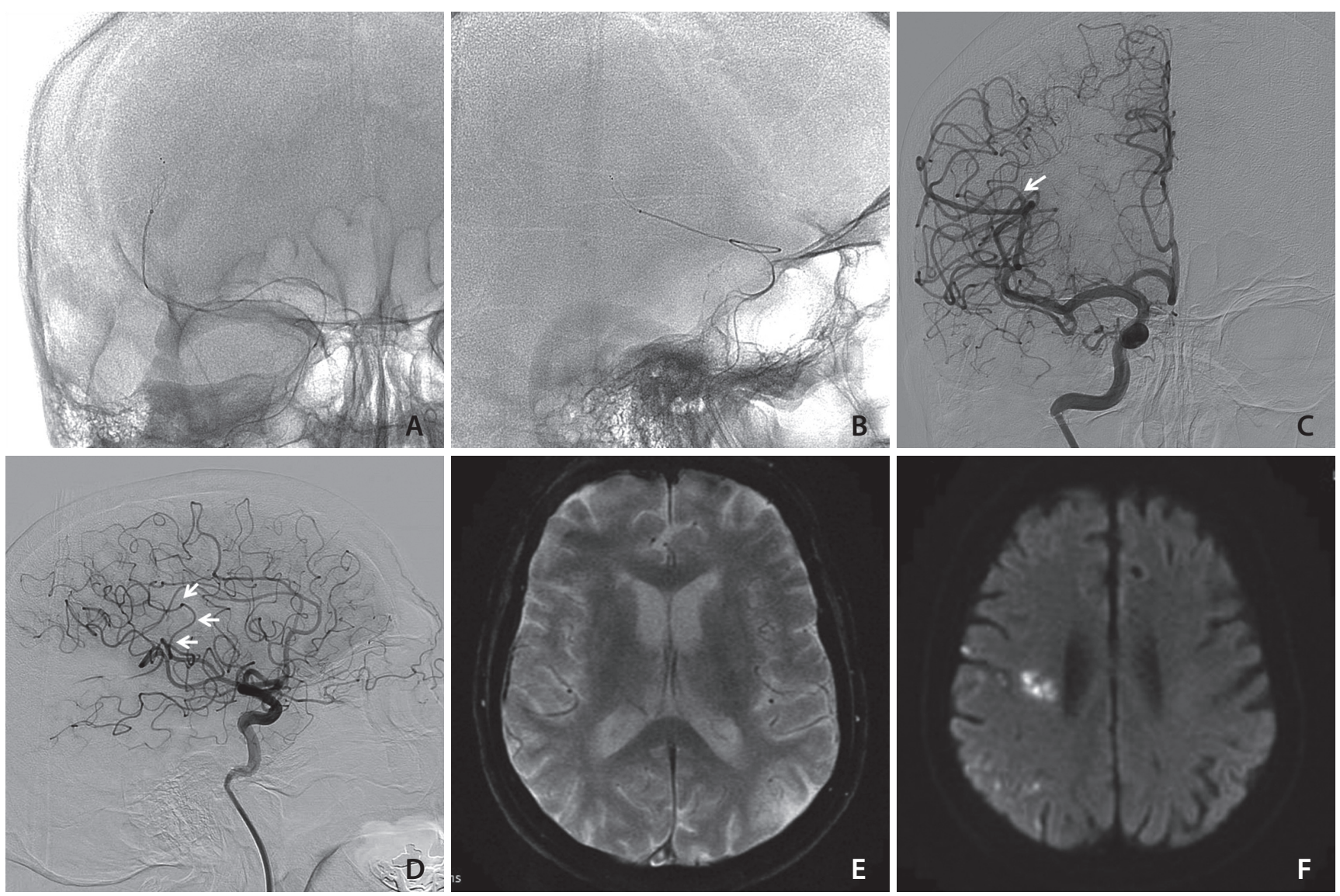

Fig. 2. (A, B) Antero-posterior and lateral fluoroscopy showing the semi-deployed "Baby" Trevo stent retriever in right precentral artery across the occluded segment, with the proximal half of the stent retriever still captured within the microcatheter. (C, D) Post thrombectomy right ICA angiogram showing revascularization of the occluded precentral artery (arrows). (E) Gradient echo MRI and (F) diffusion weighted image 72 hours after thrombectomy demonstrating no evidence of subarachnoid haemorrhage, and foci of diffusion restriction corresponding to distal emboli without a large wedge cerebral infarction. ICA, internal carotid artery; MRI, magnetic resonance imaging. 

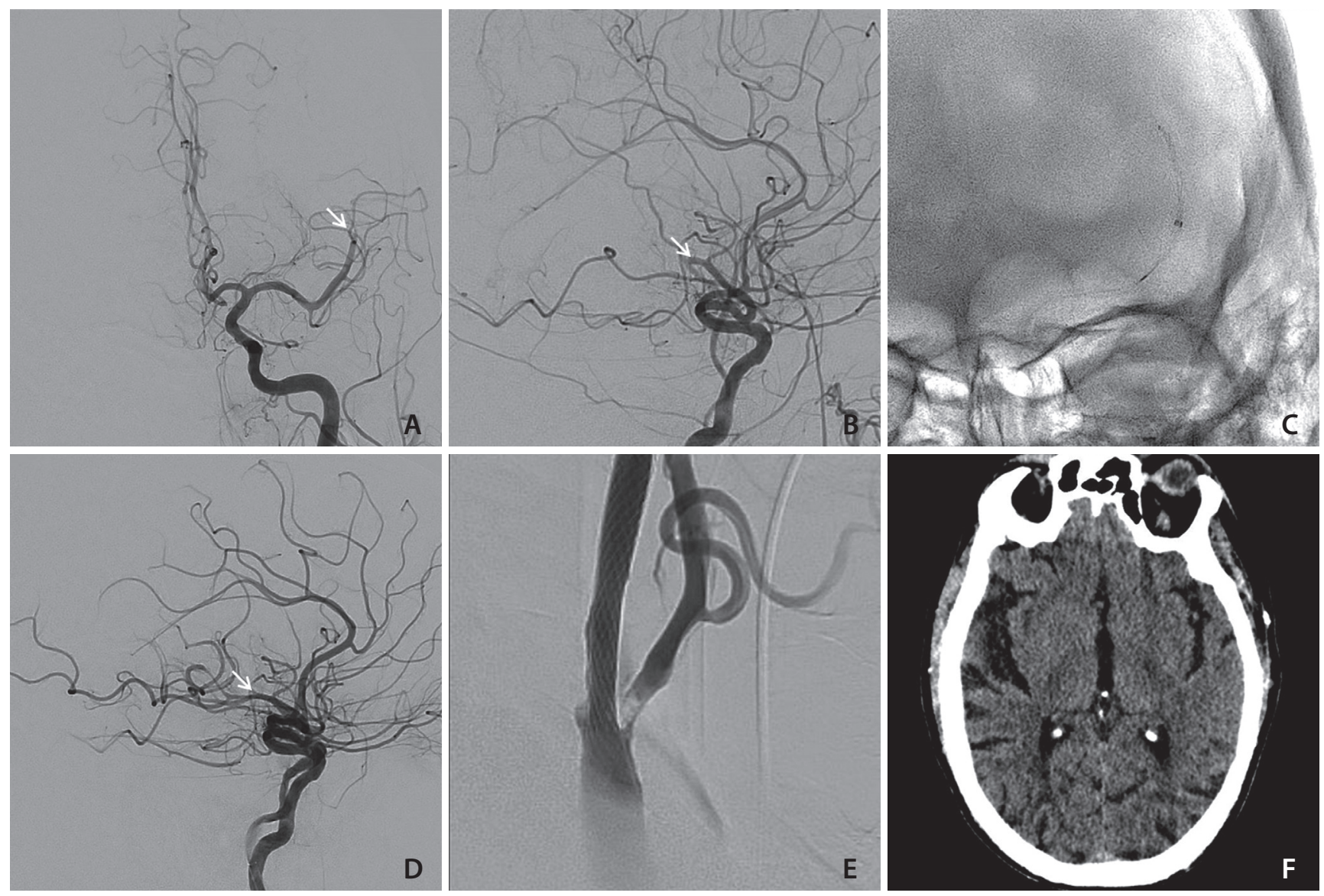

Fig. 3. (A, B) Antero-posterior and lateral view of left ICA angiogram, demonstrating the M2/3 branch occlusion (white arrow); (C) anterio-posterior fluoroscopic image showing the semi-deployed baby Trevo stent retriever, with the proximal half of the stent still restrained within the microcatheter. (D) Lateral ICA angiogram after first pass of thrombectomy demonstrating TICI 3 reperfusion and reopening of previous occluded vessel (white arrow). (E) Carotid bifurcation stenosis treated with stenting and angioplasty. (F) Brain CT 24-hours after thrombectomy showing no significant cerebral ischemia or hemorrhagic complication. ICA, internal carotid artery; TICl, treatment in cerebral infarction; CT, computed tomography.

We therefore elected to deploy half of the working length of the "Baby Trevo" stentriever in the precentral artery with just adequate length to engage the thrombus, avoiding unnecessary length of stent open across the sharp angles of the vessel. The thrombus was successfully removed with one pass and no hemorrhagic complications were encountered in both cases. A similar concept was first described by Imahori et al. ${ }^{7}$ in 14 cases of M1 or M2 occlusion using larger stent retrievers if the blood clot is short.

Aspiration thrombectomy with smaller reperfusion catheters is an alternative therapy for distal occlusions. Premat et al. ${ }^{8}$ reported good navigability of the 3 max reperfusion catheter (Penumbra Inc., Alameda, CA, USA) in M3 branches and treated six $\mathrm{M} 3$ occlusions. However, the reperfusion rate by aspiration alone seems to be lower than stent retrievers for small vessel occlusions, especially in those just distal to an angulated vessel segment. ${ }^{9}$ Stent retrieval technique also appears to have a higher first pass reperfusion rate as a standalone first-line approach to ICA/M1/M2 occlusions. ${ }^{10}$ The current case report suggests that semi-deployment of small stent retriever may be a safe and effective treatment strategy for distal occlusions.

\section{CONCLUSION}

For acute distal occlusions in small but functionally eloquent vessels such as M2/3 branches supplying the motor cortex, mechanical thrombectomy with small calibre stentrievers is reasonable and feasible. Semi-deployment of the stent retriever in small and tortuous vessels to reduce radial and tractional forces on the vascular wall can potentially avoid 
hemorrhagic complication associated with these distal thrombectomies.

\section{REFERENCES}

1. Powers WJ, Rabinstein AA, Ackerson T, Adeoye OM, Bambakidis NC, Becker K, et al. 2018 guidelines for the early management of patients with acute ischemic stroke: a guideline for healthcare professionals from the American Heart Association/American Stroke Association. Stroke 2018;49:e46-e110

2. Saber H, Narayanan S, Palla M, Saver JL, Nogueira RG, Yoo AJ, et al. Mechanical thrombectomy for acute ischemic stroke with occlusion of the M2 segment of the middle cerebral artery: a meta-analysis. J Neurointerv Surg 2018;10:620-624

3. Tan IY, Demchuk AM, Hopyan J, Zhang L, Gladstone D, Wong $\mathrm{K}$, et al. CT angiography clot burden score and collateral score: correlation with clinical and radiologic outcomes in acute middle cerebral artery infarct. AJNR Am J Neuroradiol 2009;30:525531

4. Fanous AA, Siddiqui AH. Mechanical thrombectomy: stent retrievers vs. aspiration catheters. Cor et Vasa 2016;58:e193-e203

5. Haussen DC, Lima A, Nogueira RG. The Trevo XP 3×20 mm retriever ('Baby Trevo') for the treatment of distal intracranial occlusions. J Neurointerv Surg 2016;8:295-299

6. Machi P, Jourdan F, Ambard D, Reynaud C, Lobotesis K, Sanchez $M$, et al. Experimental evaluation of stent retrievers' mechanical properties and effectiveness. J Neurointerv Surg 2017;9:257-263

7. Imahori T, Tanaka K, Koyama J, Arai A, Shiomi R, Iwahashi H, et al. Mechanical thrombectomy using the Trevo ProVue in 50 consecutive patients with anterior circulation stroke: a single-center experience after approval of the stent retriever in Japan. Neurol Med Chir (Tokyo) 2017;57:128-135

8. Premat K, Bartolini B, Baronnet-Chauvet F, Shotar E, Degos V, Muresan $P$, et al. Single-center experience using the 3MAX reperfusion catheter for the treatment of acute ischemic stroke with distal arterial occlusions. Clin Neuroradiol 2018;28:553-562

9. Kim YW, Son S, Kang DH, Hwang YH, Kim YS. Endovascular thrombectomy for M2 occlusions: comparison between forced arterial suction thrombectomy and stent retriever thrombectomy. J Neurointerv Surg 2017;9:626-630

10. Tsang COA, Cheung IHW, Lau KK, Brinjikji W, Kallmes DF, Krings T. Outcomes of stent retriever versus aspiration-first thrombectomy in ischemic stroke: a systematic review and meta-analysis. AJNR Am J Neuroradiol 2018;39:2070-2076 